\title{
Parametric Study of Greitzer's Instability Flow Model Through Compressor System Using the Taguchi Method
}

\author{
Eddie Yin-Kwee Ng, ${ }^{*}$ Ningyu Liu, and Shin Yi Tan \\ College of Engineering, School of Mechanical \& Production Engineering, \\ Nanyang Technological University, 50, Nanyang Avenue, Singapore 639798
}

The occurrence of stall and surge is caused by instability of the flow through the compressor system. These two phenomena often result in serious mechanical problems for the compressor. The scope of this article includes a review and parametric study on the characteristics of stall and surge and their mathematical modeling.

In this article, Greitzer's B-parameter model is applied for analyzing the stall and surge characteristics. Based on this model, a total of four parameters- $B, G, K$, and $L_{C}$-in the model are highlighted in order to establish the influence of each parameter on the system. Parameters $B, G$, and $K$ are all dimensionless constants while $L_{C}$ is the effective length of the compressor duct.

Here, the governing equations of stall and surge behavior are solved numerically using fourth-order Runge-Kutta method. The Taguchi method is then used to analyze the results generated to obtain the extent of effects of the parameters on the system by varying the parameters in a series of combinations. Finally, a thorough analysis is carried out on the results generated from the Taguchi method and the graphs. It is found that parameter $K$ is the deciding factor in the position of the cross point whereas parameter $B$ is the most important one in changing the length of time needed for the compressor to reach its steady state.

Keywords Compressor system, Stall, Surge, Taguchi method, Greitzer's B-parameter model

\section{INTRODUCTION}

There are two types of instabilities that could be encountered in axial compressor systems: The rotating stall and surge.

Received 5 June 2003; in final form 10 September 2003.

*Correspondence author. Tel.: (065)6790-4455, Fax: (065)67911859. E-mail: mykng@ntu.edu.sg
Both types of instability have damaging consequences to the compressor.

Rotating stall causes the compressor to operate with extremely low frequencies, resulting in an excessive high internal temperature that has an adverse effect on blade life. Surge can cause severe problems such as excessive built-up of pressure at the inlet and cyclic loading on compressor mounting. If the instability occurs, the blades might fail to produce required loading, and the engine might sustain catastrophic damage. To understand and avoid compressor stall and surge, it is important to know the characteristics of these two phenomena.

The surge and rotating stall are serious problems for the axial compressor system. As their consequences are different, it is useful to be able to predict which of these problems will occur for a given situation.

To know which will occur in a particular situation, the detail measurement and theoretical analysis have been taken during the stall inception processes (McDougall et al., 1990; Day, 1993; Wilson and Freeman, 1993). The studies for the stall inception confirmed that surge in the axial compressor is initiated by rotating stall (Jaw et al., 1999). Thus, it should be possible to inhibit the onset of surge by suppressing the occurrence of rotating stall.

In this area, the active control techniques have achieved a great success in suppressing the fluid dynamic instabilities that lead to stall and surge (Jaw et al., 1999; Day, 1993). On the contrary, the study of active control techniques is helpful in understanding the stall inception process and the detail flow condition in compressors during rotating stall or surge (Jaw et al., 1999; Day, 1993, 1994; Greitzer, 1976, 1980).

Working to understand the flow condition and compressor performance, the effective analysis and modeling can be traced back to the works of Greitzer in 1976, Moore and Greitzer (1986), Greitzer and Moore in 1986, and others. Among these works, Greitzer's B-parameter model (Greitzer, 1976) is well known and effective for analyzing the stall and surge characteristics. It is meaningful to study and further understand 
this model using current numerical method and computation facility.

Based on Greitzer's B-parameter model, the current authors numerically solve the governing equations of stall and surge behavior using the fourth-order Runge-Kutta method. Then using the Taguchi method (Taguchi, 1986, 1993) we analyze the results generated to obtain the extent of effects of the parameters on the system by varying the parameters in a series of combinations. Finally, a thorough analysis is done and the conclusion about the deciding factor for compressor characteristics is worked out.

\section{MATHEMATICAL MODEL}

Greitzer (1976) set up a mathematical model to analyze the characteristics of stall and surge. This model maps out not only the compressor but also the whole compressor system and may be used to predict whether a stall or a surge will occur at stall limit.

As shown in Figure 1, a compressor system normally consists of the following components: a compressor, an inlet annular duct, a plenum, and a throttle in an exit duct whose diameter is much smaller than that of the plenum.

Applying the equations of motions to the these components of the compressor system, we can obtain

$$
\begin{aligned}
\frac{d \dot{\tilde{m}}_{C}}{d \tilde{t}} & =B(\tilde{C}-\Delta \tilde{P}) \\
\frac{d \dot{\tilde{m}}_{T}}{d \tilde{t}} & =\frac{B}{G}\left(\Delta \tilde{P}-K \dot{\tilde{m}}^{2}\right) \\
\frac{d \Delta \tilde{P}}{d \tilde{t}} & =\frac{1}{B}\left(\dot{\tilde{m}}_{C}-\dot{\tilde{m}}_{T}\right) \\
\frac{d \tilde{C}}{d \tilde{t}} & =\frac{1}{\tilde{\tau}}\left(\tilde{C}_{S S}-\tilde{C}\right)
\end{aligned}
$$

Three non-dimensional parameters are also included in these equations. They are

$$
B=\frac{U}{2 a} \sqrt{\frac{V_{P}}{A_{C} L_{C}}} ; \quad G=\frac{L_{T} A_{C}}{L_{C} A_{T}} ; \quad K=\frac{A_{C}^{2}}{A_{T}^{2}} .
$$

Here $\tilde{\tau}$ is the non-dimensional time lag, $\omega$ is the Helmholtz frequency, $\Delta P=P_{P}-P$ is the pressure difference across the duct, $C$ is the pressure rise across the compressor, $\dot{m}$ is the mass

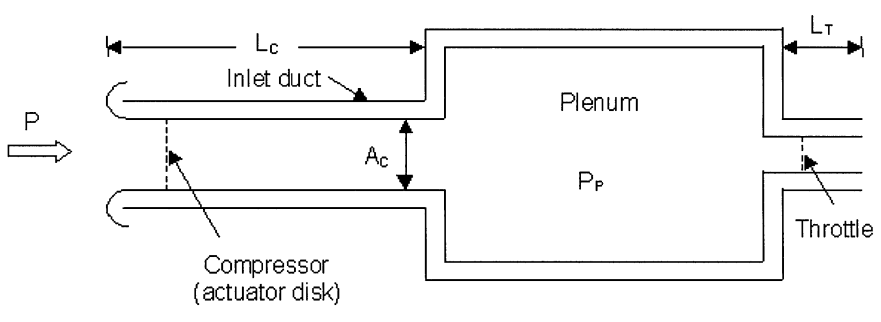

FIGURE 1

Simplified diagram of compression system [1]. flow rate, $L$ and $A$ are the length and area, and $\tau$ and $C_{S S}$ are the compressor flow field time constant and the steady-state measured compressor curve, respectively. $N$ is the time for some number of rotor revolutions, the subscripts $C, P$, and $T$ are the compressor duct, plenum, and throttle duct, respectively, the subscript $O$ is initial non-dimensionalized value at surge point (or stall point), and the top cap $\sim$ is the non-dimensionalized quantity. The non-dimensional parameters are $\rho U A_{C}$, the pressure difference variable $0.5 \rho U^{2}$, and the time variable $1 / \omega$.

Equation (1) is the non-dimensionalized 1-D governing equation for the compressor system at transient state, which is the so-called Greitzer's B-parameter model. The dimensionless $B$-parameter represents geometrical and physical parameters of system. Low values of $B$ favor rotating stall characteristic and high values of $B$ favor surge like oscillation whereas similar values of $B$ give similar flow behavior. For high values of $B$ (implying very large plenum volume) the plenum pressure remains high as the compressor starts to stall. The resulting large pressure difference across the compressor duct is enough to rapidly decelerate the flow. For low values of $B$ (small volumes), the plenum pressure drops quickly compared with the time it takes to reverse the flow in the compressor ducting. There is then an insufficient pressure difference tending to drive the flow backward through the compressor and the operating point settles down on the rotating stall characteristic. It is indeed a critical $B^{*}(\approx 0.7)$ which separates systems that exhibit surge and those that exhibit rotating stall (i.e., transient behavior after initial instability depends on $B$ ). In short, surge will occur whenever the release of the stored energy of the high-pressure fluid is enough to overcome the inertia of the fluid in the compressor ducting (i.e., we can view $B$ parameter as a ratio of stored energy to work necessary to overcome inertia forces). Parameter $G$ is a volume ratio (of cross product of effective length of throttle and compressor through flow area to effective length of compressor ducting and throttle through flow area, $G$ can be interpreted as an pointer on flow continuity). Parameter $K$ is the through flow area ratio of compressor to throttle (high $K$ values imply throttling capacity is small).

To sum up, Greitzer's model is instructive since it stresses the need to correctly model the capacity of a system to store energy, and the need to correctly account for the inertia of the fluid in the ducting. This is vital in the sense that whether or not a system will surge is not a property of the compressor, but of the compressor and the system of which it is part. Three parameters, $B, G$, and $K$, are used in this model. Together with the characteristic length $L_{C}$, these four parameters will be investigated in a series of combinations to analyze the effects of each parameter on the whole compressor system using the Taguchi method.

\section{NUMERICAL METHODS}

In this article, the characteristics of stall and surge of a particular compressor system are analyzed using the numerical method. Thereafter, the Taguchi method is applied to perform a parametric optimization for compressor identification, and the 
fourth-order Runge-Kutta method is used to solve the governing Eq. (1).

An empirical compressor pressure rise, $\tilde{C}_{S S}$, is formulated according to Greitzer (1976). The initial conditions, at $t=0$, are taken as:

(1) $\Delta \tilde{P}=\tilde{C}=\tilde{C}_{O}$

(2) $\dot{\tilde{m}}_{C}=\dot{\tilde{m}}_{T}=\dot{\tilde{m}}_{O}$

where $\tilde{C}_{O}$ and $\tilde{m}_{O}$ are the initial non-dimensionalized compressor pressure rise and mass flow.

The Taguchi method $(1986,1993)$ is a very efficient tool for analyzing and developing high quality products at a low cost. Traditionally, one tends to change only one variable of an experiment at a time. The strength of the Taguchi technique is that one can change many variables at the same time and still retain control of the experiment.

In the Taguchi method, the experiments shall be conducted by using a series of combinations from the different factors and levels of the input design variables. This may not be always feasible in a real experiment due to many practical difficulties in conducting the experiment such as a very high temperature/pressure study, hazardous conditions, cost, time, etc. However, the numerical experiments have the great advantage in the cases of combining the Taguchi method and numerical technique.

In the Taguchi method, the grouping of the iterations from the various parameters of the input design variables is called an orthogonal array. Each parameter is referred to as a factor and each different value for each factor is termed as a level. The number of combinations is obtained using the following formula:

Number of combinations $=(\text { Number of levels })^{\text {Number of factors }}$

Hence, for tabulations involving 3 factors and 3 levels as done in this article, number of combinations $=3^{3}=27$.

In the present parametric research, we solve the governing Eq. (1) by using orthogonal arrays, $L_{27}\left(3^{3}\right)$, to identify the factor/variable that has the most influence on the rotating stall/surge so as to minimize it in the actual operation of the axial compressor.

From Greitzer (1976), the most critical parameter will be $B$ with $G$ having only minor effect. Therefore $G$ is kept constant at the value of 0.36 . However in the present work, $G$ will be analyzed systematically using the Taguchi method to verify the above assumption. Hence the values of $G$ will be set at three levels with $0.16,0.36$, and 0.56 , respectively.

In Greitzer's paper (1976), surge will occur when the critical value of $B$ is approximately 0.7 . Hence in the current study, the value of $B$ used will be kept below 0.7 to avoid the occurrence of surge with the three values being $0.50,0.55$, and 0.60 , respectively.

As for the other two parameters, $K$ and $L_{C}$, their effects on the system are not mentioned in the previous paper. Therefore in the current work, these effects on the system will also be ana-
TABLE 1

Case 1: Factors and Levels for Parameters $B, G$, and $K$

\begin{tabular}{lccc}
\hline & \multicolumn{3}{c}{ Levels } \\
\cline { 2 - 4 } Factors & 1 & 2 & 3 \\
\hline$B$ & 0.50 & 0.55 & 0.60 \\
$G$ & 0.16 & 0.36 & 0.56 \\
$K$ & 5.32 & 5.52 & 5.72 \\
\hline
\end{tabular}

lyzed to complete the whole spectrum of parametric study. The two parameters will be categorized into two separate cases with parameters $B$ and $G$ as listed in Tables 1 and 2. Upon executing the program, the results are compiled and included/tabulated in Tables 3 and 4.

As shown in Tables 3 and 4, there are columns for values of Ave $X$ and Ave $Y$, which are calculated from the average of the $X$ and $Y$ coordinates of the cross point $(X, Y)$ of the compressor curve when it stabilizes to a stationary point.

The values for Ave Diff $M$ and Ave Diff $P$ are obtained similarly. Firstly, the maximum and minimum values of the instantaneous pressure rise $P$ and the compressor axial velocity parameter $M$ are recorded in the process of prediction. Then the difference between these two ranges of values, Diff $M$ and Diff $P$, are calculated and averaged subsequently. The averages of the values $X, Y, \operatorname{Diff} M$, and Diff $P$ are calculated according to their levels. This system of calculation will apply to all three levels of the three parameters for the two cases.

Last but not least, the final results indicating the effects of the parameters on the compressor system are tabulated. Referring to the last four columns of Tables 3 and 4, represented by $R(X) \%$, $R(Y) \%, R($ Diff $M) \%$, and $R(D$ iff $P) \%$, they are the percentage values signifying the importance of the parameters. The higher the percentage value, the greater the effect the parameter has on the system.

Figures 2 to 9 are plotted from the data files generated upon the execution of the program. The graphs drawn will be divided into two categories. In the first category, the graphs (Figures 2 $5)$ will focus on the changes in the cross point $(X, Y)$. In the second category, the changes in the "diameters of oscillations" (Diff $M$ and Diff $P$ ) will be investigated (Figures 6-9). In each figure, only one parameter is varied accordingly to their three levels, and the other parameters will be set at its original value as

TABLE 2

Case 2: Factors and Levels for Parameters $B, G$, and $L_{C}$

\begin{tabular}{lccc}
\hline & \multicolumn{3}{c}{ Levels } \\
\cline { 2 - 4 } Factors & 1 & 2 & 3 \\
\hline$B$ & 0.50 & 0.55 & 0.60 \\
$G$ & 0.16 & 0.36 & 0.56 \\
$L_{C}$ & 1.36 & 1.46 & 1.56 \\
\hline
\end{tabular}


TABLE 3

Case 1: Results for Effects of Parameters $B, G$, and $K$ on the Compressor System

\begin{tabular}{lcccccccc}
\hline & Ave $X$ & Ave $Y$ & Ave Diff $M$ & Ave Diff $P$ & $R(X) \%$ & $R(Y) \%$ & $R($ Diff $M) \%$ & $R($ Diff $P) \%$ \\
\hline$B$ & 0.414529 & 0.951680 & 0.042722 & 0.054561 & 0.142237 & 0.252039 & 5.360667 & 10.329344 \\
& 0.415944 & 0.954201 & 0.062836 & 0.093696 & & & & \\
& 0.415951 & 0.954045 & 0.096328 & 0.157855 & & & & \\
$G$ & 0.414513 & 0.951581 & 0.074464 & 0.114550 & 0.144583 & 0.261614 & 1.216133 & 2.111956 \\
& 0.415952 & 0.954147 & 0.065120 & 0.098131 & & & & \\
& 0.415959 & 0.954197 & 0.062302 & 0.093431 & & & & \\
$K$ & 0.426744 & 0.972833 & 0.060423 & 0.095681 & 2.253574 & 3.841621 & 1.551422 & 1.558044 \\
& 0.415472 & 0.952675 & 0.065525 & 0.099169 & & & & \\
& 0.404208 & 0.934417 & 0.075937 & 0.111262 & & & & \\
\hline
\end{tabular}

proposed in the previous study, $B=0.60, G=0.36, K=5.52$, and $L_{C}=1.46$.

\section{RESULTS AND DISCUSSION}

The analysis of the influences of parameters $B, G, K$, and $L_{C}$ will be based on the results obtained upon performing the Taguchi method on these four parameters. The results can be found in the last four columns of Tables 3 and 4, respectively. As seen from the values obtained, they are presented in percentage values. This implies that the higher the percentage value, the higher the influence of the parameter.

\section{Analysis for Case 1: With Parameters $B, G$, and $K$}

Referring to Table 3 for the results of the parameters $B, G$, and $K$, it can be seen that parameters $G$ and $K$ affect the coordinates of the cross point $(X, Y)$ most with parameter $B$ having the least influence. It can be observed that the coordinates of the cross point $(X, Y)$ shift slightly upon the variation of the values of $K$. As $K$ increases, both the values of the $x$ - and $y$ coordinate of the cross point decrease (Figure 2). The values of the $x$ - and $y$-coordinate will shift an overall of $2.25 \%$, and $3.84 \%$, respectively when $K$ is changed, as indicated in Table 3 .
On the other hand, from Table 3 , the $x$ - and $y$-coordinate will move an overall of $0.145 \%$ and $0.262 \%$, respectively, when $G$ changes. These small changes cannot even be observed in Figure 3. This implies that $K$ has a greater effect on the position of cross point $(X, Y)$. The smaller influence of $B$ can also be noticed from Figure 4.

From Table 3, with a percentage of about $5 \%$ and $10 \%$ influence on the values of Diff $M$ and Diff $P, B$ is considered the deciding factor. Hence it can be seen from Figure 6 that upon increasing the value of $B$ from 0.5 to 0.6 , the diameter of oscillation increases significantly from an average of 0.043 to 0.096 and 0.055 to 0.158 , respectively for both of the two axes. Compared with $B, K$ has a smaller effect on the diameter of oscillation,.

On the other hand, looking at Figure 7, if only $G$ is increased with the rest of the parameters remaining as constants, the increase in the diameter of oscillation is very small. When $G$ increases from 0.16 to 0.56 , the average values for Diff $M$ and Diff $P$ for both of the two axes decrease from 0.0745 to 0.062 and 0.115 to 0.093 , respectively (Table 3). This results in an average value of $1.22 \%$ and $2.11 \%$. Similarly for parameter $K$, as shown in Table 3 and Figure 8, there is a small average value of $1.55 \%$ and $1.56 \%$. When compared with the percentage values for $B$, these values are much smaller.

TABLE 4

Case 2: Results for Effects of Parameters $B, G$, and $L_{C}$ on the Compressor System

\begin{tabular}{ccccccccc}
\hline & Ave $X$ & Ave $Y$ & Ave Diff $M$ & Ave Diff $P$ & $R(X) \%$ & $R(Y) \%$ & $R($ Diff $M) \%$ & $R($ Diff $P) \%$ \\
\hline$B$ & 0.415453 & 0.952757 & 0.037022 & 0.045106 & 0.002900 & 0.034756 & 5.959733 & 11.358556 \\
& 0.415459 & 0.952761 & 0.063036 & 0.094409 & & & & \\
& 0.415482 & 0.952413 & 0.096619 & 0.158691 & & & & \\
$G$ & 0.415422 & 0.952525 & 0.068912 & 0.105351 & 0.007220 & 0.023289 & 0.646244 & 1.130422 \\
& 0.415479 & 0.952649 & 0.065314 & 0.098809 & & & & \\
& 0.415494 & 0.952757 & 0.062450 & 0.094047 & & & & \\
$L_{C}$ & 0.415468 & 0.952743 & 0.057156 & 0.079110 & 0.001674 & 0.022910 & 1.683922 & 4.082233 \\
& 0.415472 & 0.952675 & 0.065525 & 0.099164 & & & & \\
& 0.415455 & 0.952514 & 0.073996 & 0.119932 & & & & \\
\hline
\end{tabular}




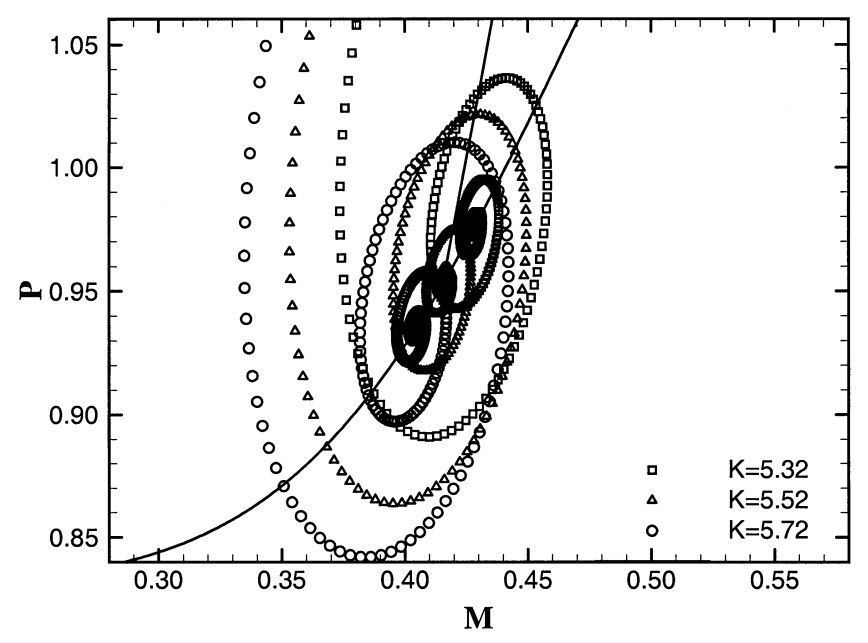

FIGURE 2

Effect of changes of $K$ on the cross point $(X, Y)$.

\section{Analysis for Case 2: With Parameters $B, G$, and $L_{C}$}

Similar analysis is done for the second group of parameters $B, G$, and $L_{C}$. Referring to Table 4, the last four columns complete the final values of the tabulation. As shown in the two columns for the percentage values $R(X)$ and $R(Y)$ in affecting the coordinates of the cross point $(X, Y)$, it can be deduced that parameters $G$ and $B$ are the two prime factors while $L_{C}$ is the least important. The locations of the cross point $(X, Y)$ when the values of $G, B$, and $L_{C}$ change are shown in Figures 3, 4, and 5 , respectively.

When $B$ increases, the $x$ - and $y$-coordinate of the cross point increase very little with changes at about $0.003 \%$ and $0.035 \%$. Similarly for the parameter $G$ when its value is increased, the percentage increase for both coordinates is very small, being $0.007 \%$ and $0.023 \%$, respectively. Though parameters $B$ and $G$ show some influence in affecting the position of the cross point $(X, Y)$, their effects are very small.

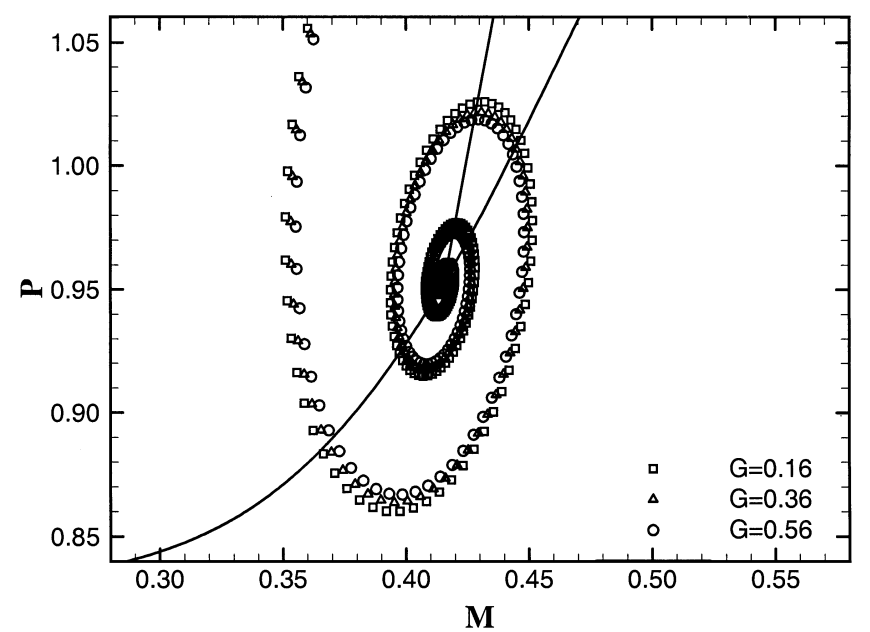

FIGURE 3

Effect of changes of $G$ on the cross point $(X, Y)$.

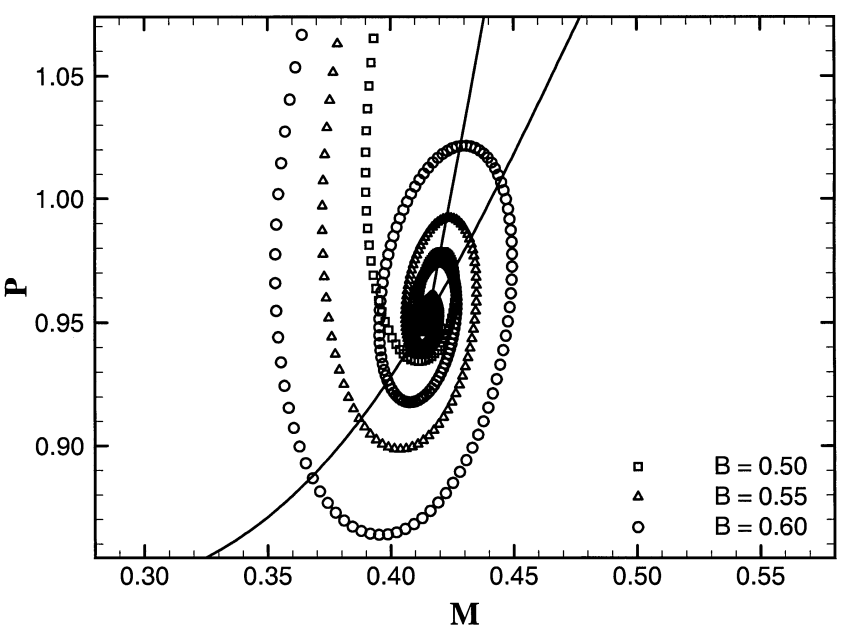

FIGURE 4

Effect of changes of $B$ on the cross point $(X, Y)$.

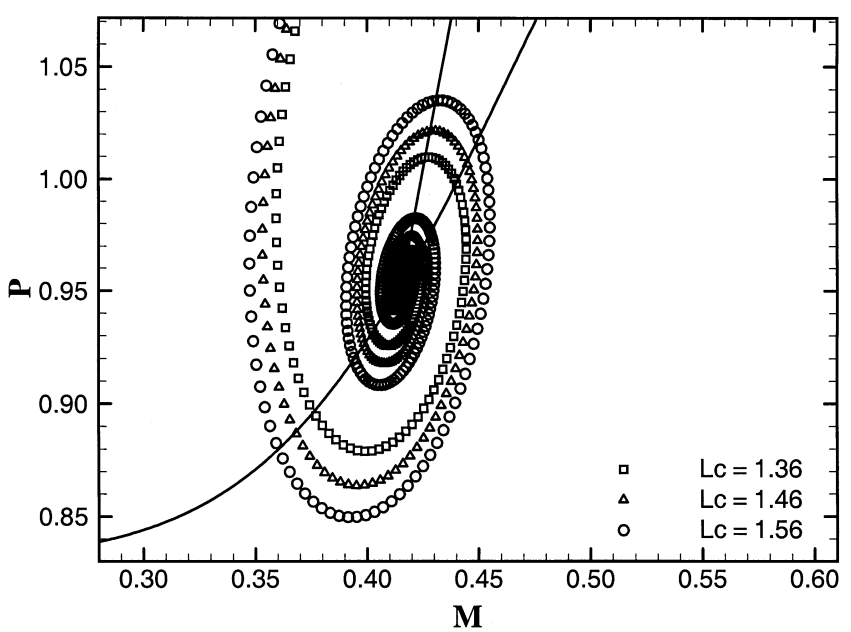

FIGURE 5

Effect of changes of $L_{C}$ on the cross point $(X, Y)$.

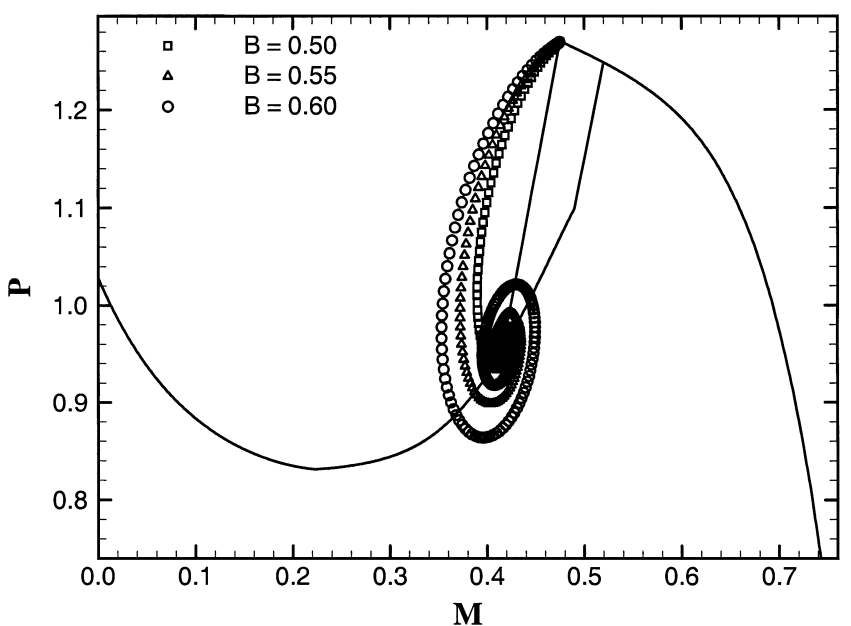

FIGURE 6

Effect of changes of $B$ on the diameter of oscillation. 


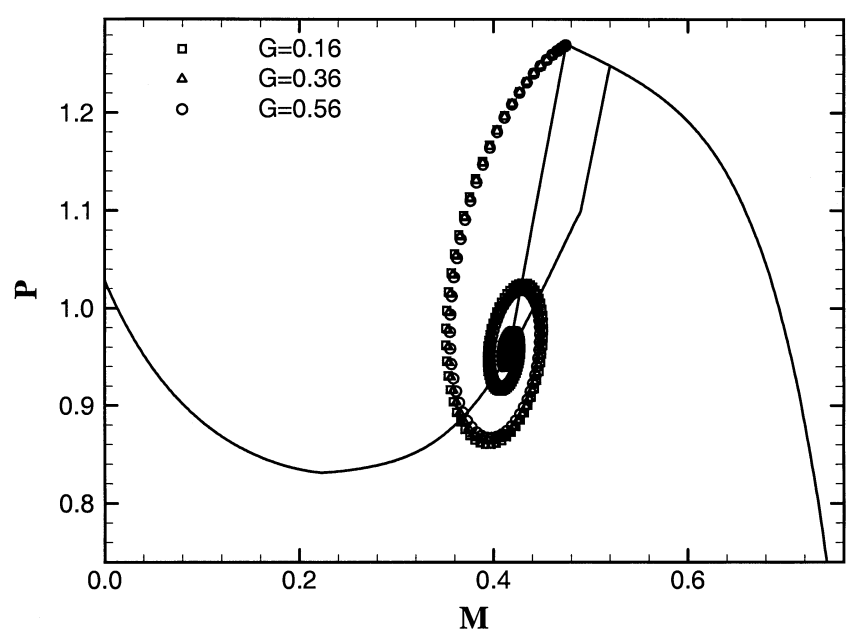

FIGURE 7

Effect of changes of $G$ on the diameter of oscillation.

The last two columns of Table 4 indicate the percentage values of the diameter of oscillation for each parameter. It can be seen that parameter $B$ has the highest value followed by parameter $L_{C}$ and then parameter $G$. The effects of parameters $B$ and $G$ on the diameter of oscillation have been analyzed in Case 1 with Figures 6 and 7.

With reference to Figure 9, the diameter of oscillation increases with an increase in the value of $L_{C}$. Diff $M$ and Diff $P$ increase from an average of 0.057 to 0.074 and 0.079 to 0.120 . This indicates an increase of $1.68 \%$ and $4.08 \%$, respectively. However, when compared with the percentage increase of $B$, those effects by $L_{C}$ are still considered small. This implies that $B$ parameter actually plays a more important role in the diameters of oscillations thus affecting the time taken for the system to reach a steady state.

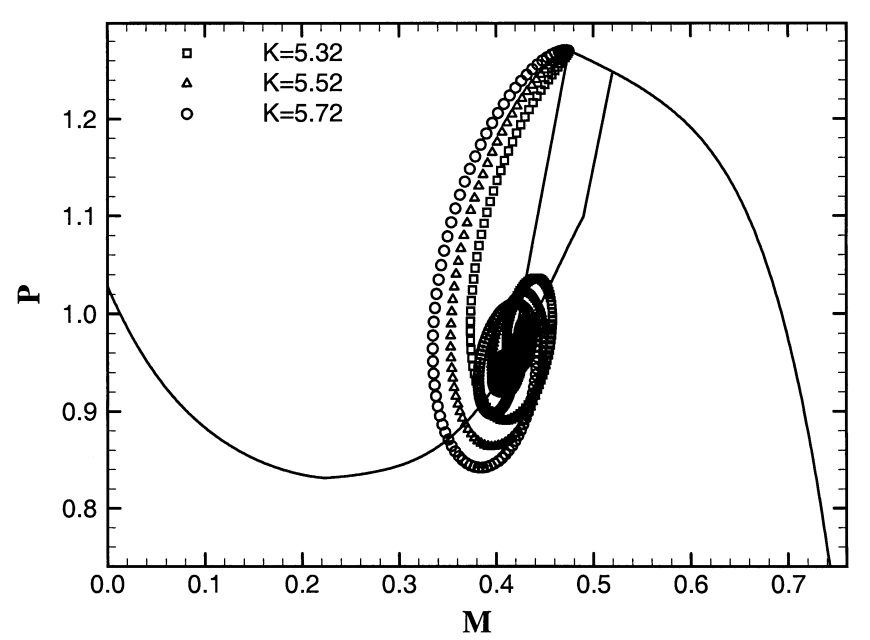

FIGURE 8

Effect of changes of $K$ on the diameter of oscillation.

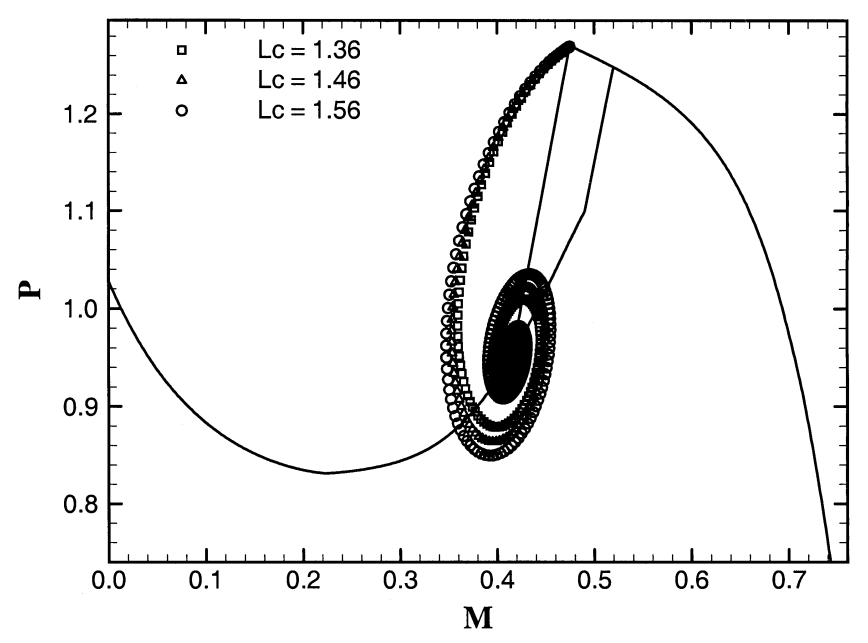

FIGURE 9

Effect of changes of $L_{C}$ on the diameter of oscillation.

\section{Summary: Parameters $B, G, K$, and $L_{C}$}

By mapping the results obtained from the two Taguchi Tables 3 and 4, a final analysis can be deduced.

It can be concluded that parameter $K$ is the most important factor in deciding the position of cross point $(X, Y)$. It is then followed by $G, B$, and $L_{C}$ in order of importance. From Tables 3 and 4, the percentage values of changes in the $x$ - and $y$-coordinates of the cross point of $K$ is the highest with values of $2.25 \%$ and $3.84 \%$ thus implying that it affects the position of the cross point the most. $L_{C}$ however, has the least influence with values at $0.0017 \%$ and $0.023 \%$, respectively.

Similarly, the analysis for the diameter of oscillation of the curve is obtainable. The most influencing factor is $B$ followed by $L_{C}, G$, and $K$. In conclusion, $B$ has the highest percentage values and contributes a significant percentage in affecting the performance of the compressor system.

\section{CONCLUSION}

In this article, after reviewing the previous work on stall and surge of an axial compressor system and their mathematical models, the Greitzer's B-parameter model is applied to study the stall and surge characteristics. Thereafter, the Taguchi method is used to further analyze the extent of the effects that the four parameters $B, G, K$, and $L_{C}$ have on the system. It is found that the Taguchi method succeeds in obtaining the important parameters that will affect the system.

The analysis on the four parameters, $B, G, K$, and $L_{C}$, has been carried out with satisfying results. It is observed that parameters $B$ and $K$ are crucial in maintaining the steady state operation of the compressor system. With much higher percentage value for the changes in Diff $M$ and Diff $P$, the parameter $B$ highly affects the range of diameters of oscillation. On the other hand, $K$ is the deciding factor in the position of the cross point with a higher percentage value in the variation of $x$ - and $y$-coordinate. 
In short, analysis on the compressor system has been done by varying the values of $B$ under the critical value $(\approx 0.7)$. In Greitzer's model (1976), analysis of the effect of $B$ on the system has been carried out to varying values of $B$ up to 5 . However, as the Taguchi method has not been carried out previously on this system, analysis involving a greater range of $B$ could be done as a further work. By varying the value of $B$ from 0.7 (critical) onwards, the behavior of the system when it reaches surge can then be investigated.

\section{ACKNOWLEDGEMENT}

This research was conducted in-line with the Republic of Air Force Singapore Industry Program.

\section{NOMENCLATURE}

$a$

A

$B$

$C$

\section{$C_{x}$}

$C_{S S}$ steady-state compressor pressure rise

$F \quad$ throttle pressure drop

G

\section{$k$}

\section{K}

\section{$L$}

speed of sound

area of the compressor duct

dimensionless parameter, $B=\frac{U}{2 a} \sqrt{\frac{V_{P}}{A_{C} L_{C}}}$

compressor pressure rise

compressor axial velocity

dimensionless parameter, $G=\frac{L_{T} A_{C}}{L_{C} A_{T}}$

polytropic exponent

dimensionless parameter, $K=\frac{A_{C}^{2}}{A_{T}^{2}}$

effective length of equivalent duct

mass flow rate

compressor axial flow coefficient, $C_{x} / U$

pressure

plenum pressure rise

compressor rotor mean radius

time

mean rotor velocity

volume

$x$ coordinates of the cross point

$y$ coordinates of the cross point

ratio of specific heats

density

compressor flow field time constant

Helmholtz frequency

\section{Subscripts}

$O \quad$ initial nondimensionalized value at surge point (or stall point)

C compressor

$P \quad$ plenum

$T$ throttle

$x \quad x$-axial direction

\section{Superscript}

$\sim$ nondimensionalized variable

\section{REFERENCES}

Day, I. J. 1993. Review of Stall Surge and Active Control in Axial Compressors, ISABE 93-7011, pp. 97-105.

Day, I. J. 1993. Stall inception in axial flow compressors. Transactions of the ASME, Turbomachinery 115:1-9.

Day, I. J. 1994. Axial compressor performance during surge. Journal of Propulsion and Power 10(3):329-336.

Greitzer, E. M. 1976. Surge and rotating stall in axial flow compressors. Part I: Theoretical compression system model. Part II: Experimental results and comparison with theory Transactions of the ASME, Journal of Engineering for Power 98:190-217.

Greitzer, E. M. 1980. Review-axial compressor stall phenomena. Transactions of the ASME, Journal of Fluids Engineering 102:134143.

Greitzer, E. M., and Moore, F. K. 1986. A theory of post-stall transients in axial compression systems. Part II: Applications. Journal of Engineering for Gas Turbines and Power 108:231-240.

Jaw, L. C. et al. (1999) A high-response high-gain actuator for active flow control. AIAA paper 99-2128.

McDougall, N. M., Cumpsty, N. A., and Hynes, T. P. 1990. Stall inception in axial compressors. Transactions of the ASME, Turbomachinery 112:116-125.

Moore, F. K., and Greitzer, E. M. 1986. A theory of post-stall transients in axial compression systems. Part I: Development of Equations. Journal of Engineering for Gas Turbines and Power 108:68-76.

Taguchi, G. 1986. Introduction to Quality Engineering: Designing Quality into Products and Processes, Japan: Asian Productivity Organization.

Taguchi, G. 1993. Taguchi on Robust Technology Development: Bringing Quality Engineering Upstream, ASME Press, New York.

Wilson, A. G., and Freeman, C. 1993. Stall Inception and Development in an Axial Flow Aero-Engine, ASME Paper 93-GT-2. 

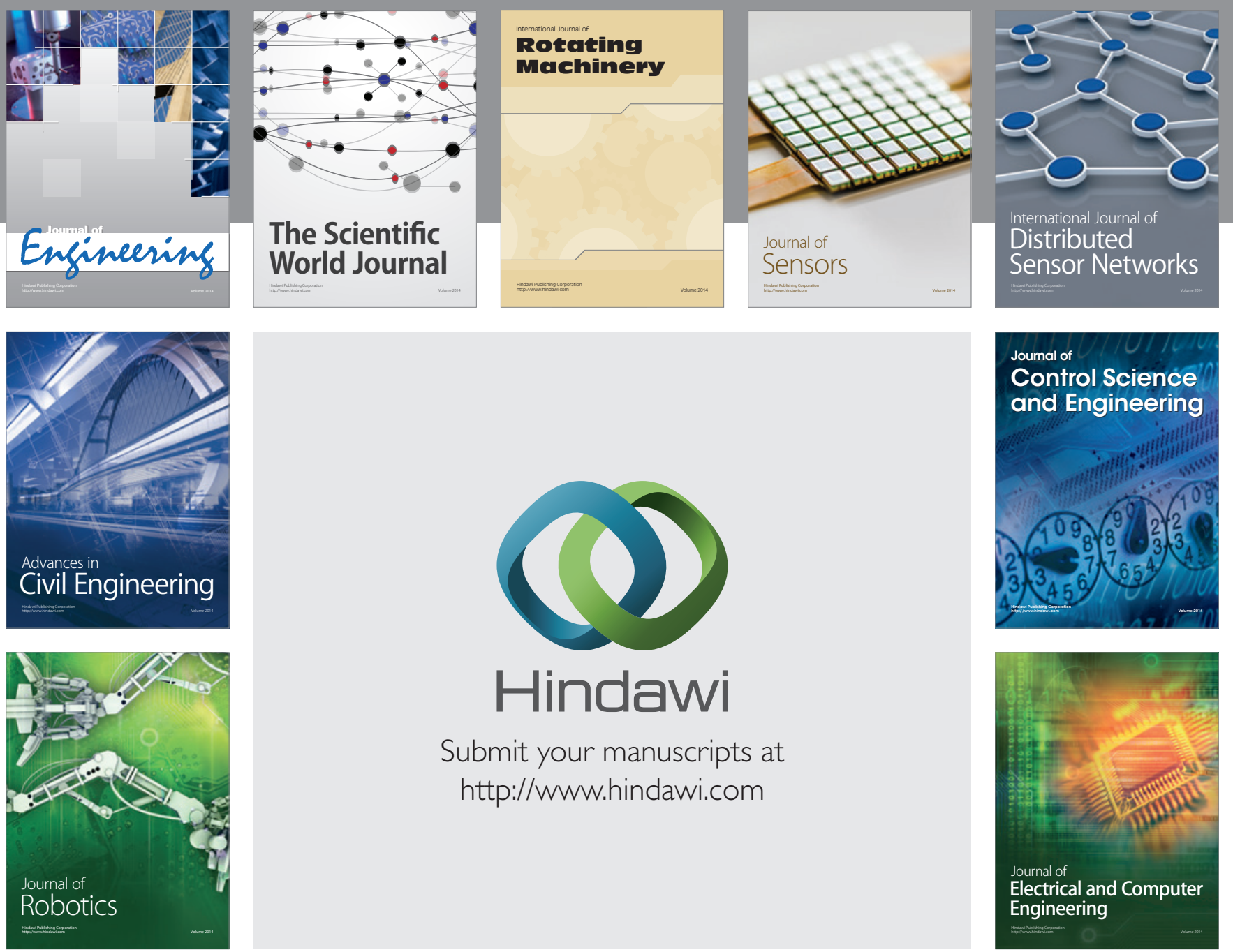

Submit your manuscripts at

http://www.hindawi.com
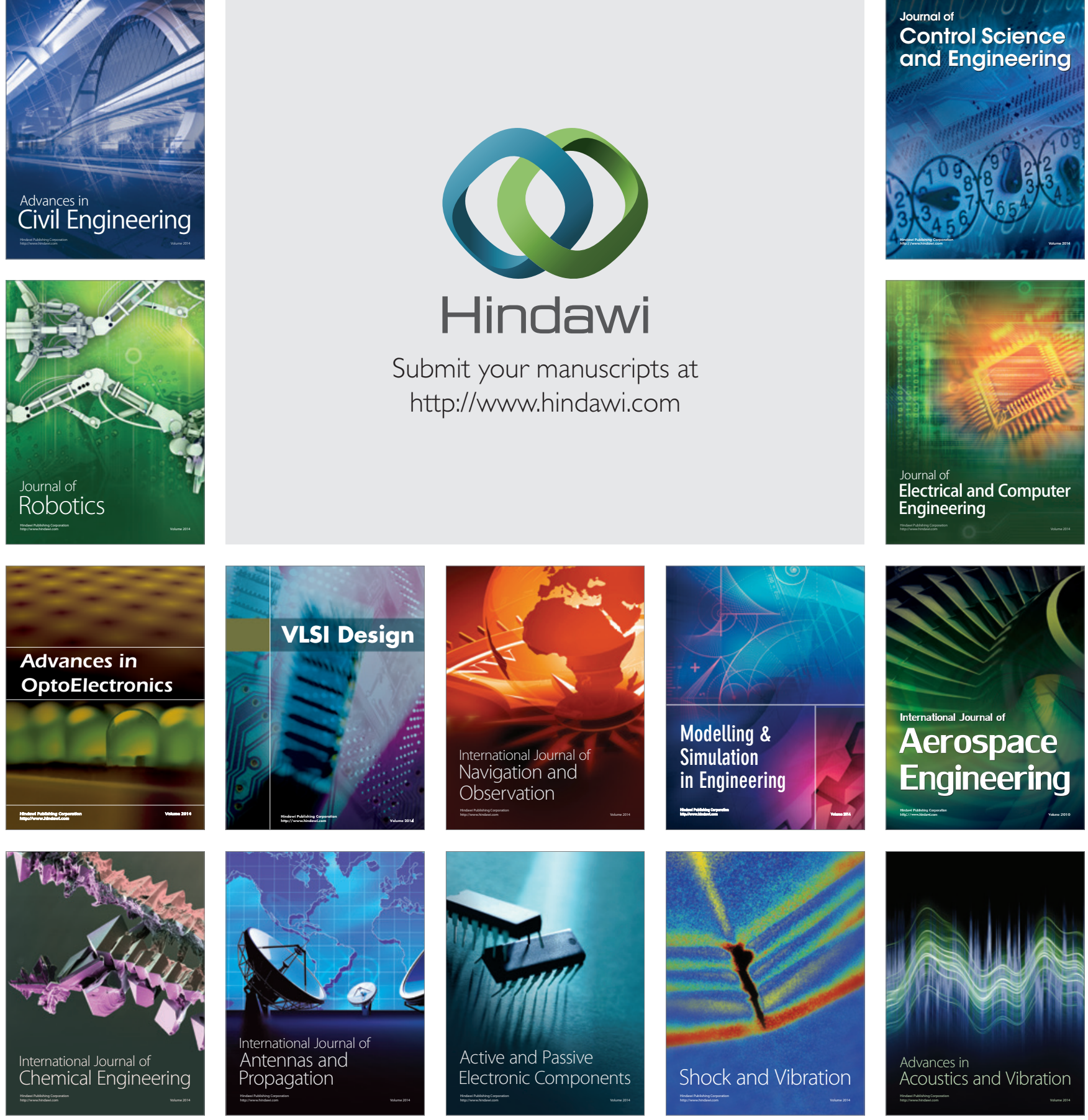